\section{Die ITA-Konzeption des BMBF - nur ein Ansatz in der deut- schen TA/TAG-Diskussion}

\author{
von Paul Fuchs-Frohnhofen, Mensch, Arbeit \\ und Technik Sell \& Partner GmbH, Aachen
}

\section{Einführung}

Aufgefordert als Ingenieur zu dem Papier „Die ITA der Gesellschaft" von Bode (Bode 2002) Stellung zu nehmen, möchte ich auf drei Aspekte meines Verständnisses von Technikfolgenabschätzung und Technikgestaltung (TA/TG) hinweisen:

- die Wertediskussion im Feld TA/TG,

- die ingenieurwissenschaftliche Sicht auf TA/TG,

- eine andere Bedeutungszuweisung als die von Bode für „ITA“ in Ingenieur- und Sozialwissenschaften.

Damit beabsichtige ich nicht, den konstruktivistischen Ansatz von Bode grundsätzlich in Frage zu stellen, sondern ich möchte deutlich machen, dass ,eine konstruktivistische Theorie ... - wie jede andere Theorie auch - (nur) eine Möglichkeit von mehreren (ist), die Welt zu beobachten" (Bode 2002, S. 38).

In einigen abschließenden Bemerkungen möchte ich auf die heutige Situation der TA in Deutschland kurz eingehen.

\section{Von der „Theorie sozialer Systeme“ zur Innovations- und Technikanalyse (ITA)}

In dem o.g. Text beruft sich Bode auf die "Theorie sozialer System" von Niklas Luhmann und geht dabei von einer ,radikalkonstruktivistischen" Position aus (Bode 2002, S.37). Aus dieser Sichtweise stellt sich für ihn „moderne Gesellschaft“ in verschiedene Funktionalsysteme wie Wirtschaft, Politik, Wissenschaft, Recht und Erziehung gegliedert dar, die er wieder jeweils als autopoietische Kommunikationssysteme bezeichnet, die füreinander „undurchdringbare Grenzen“ aufbauen (Bode 2002, S.39). Daraus folgert er (vereinfacht dargestellt), dass Politik der Möglichkeit, ihre Entscheidungen direkt umzusetzen, beraubt ist und sich deswegen um die Schaffung von Anschlussfähigkeit für die anderen Funktionalsysteme durch „Perturbation“ und „Partizipation“ bemühen muss. Perturbation bedeutet für ihn, dass Politik den ,anderen Kommunikationssystemen Bezüge für deren eigene Beobachtungen liefert", so dass mit der dem jeweiligen Funktionalsystem eigenen Kommunikation an Politik angeknüpft werden kann, während er es Partizipation nennt, wenn solche Bewusstseine mit Gedanken an die Ergebnisse politischer Kommunikation anschließen, die eigentlich einem anderen Funktionalsystem angehören.

Bezüglich seines eigentlichen Themenfeldes, der Innovations- und Technikanalyse, macht er dann die Funktionalsysteme Wirtschaft, Politik und Wissenschaft als die wesentlichen im Prozess der Innovations- und Technikgenese aus und bietet das BMBF als Intermediär an, über den durch ITA-Projekte Perturbation und Partizipation zwischen den drei genannten Funktionalsystemen organisiert werden kann.

Aus meiner Sicht ist die zugrunde liegende Überlegung seiner Ausführungen, dass nämlich moderne Gesellschaft aus Kommunikationssystemen mit „füreinander undurchdringbaren Grenzen", den Funktionalsystemen besteht, nur eine Möglichkeit, wie Wissenschaft das Phänomen einer komplexen Gesellschaft analytisch beschreiben kann. Die aus diesem Beschreibungsmodell abgeleiteten Folgerungen werden für mich für praktische Arbeit in Politik, Zivilgesellschaft, Wissenschaft, Technikentwicklung und Technikfolgenabschätzung dann hinderlich, wenn sie Kommunikations- und Beeinflussungsunfähigkeit postulieren, wo ich in praktischer Arbeit gemeinsame Kommunikationsfähigkeit und gegenseitige Beeinflussungsfähigkeit dieser Funktionalsysteme vorfinde.

Obwohl ich also einige der grundlegenden Überlegungen von Bode nicht teilen kann, gehen viele der praktischen Konsequenzen seiner theoretischen Überlegungen in die gleiche Richtung, die viele Wissenschaftler/innen, Politiker/ innen, Ingenieur/innen und andere im Bereich der von Bode als „historisches Vorgängermodell" seiner modernen ITA bezeichneten „Technikfolgenabschätzung und Technikgestaltung" seit Jahrzehnten fordern und realisieren helfen: 
- interdisziplinäre Kooperation und Kommunikation in der Wissenschaft,

- Kommunikation zwischen Politik, Wissenschaft, Wirtschaft und Zivilgesellschaft (letztere kommt bei Bode nicht vor) ${ }^{1}$

- die Betrachtung von Technikgenese als komplexen, nebenwirkungsbehafteten Prozess, der nicht nur die Technik selbst umfasst, sondern in Wechselwirkung von Technik, Wissenschaft, Wirtschaft, Zivilgesellschaft und Politik zu konkreten materiellen Ergebnissen führt, die aber wiederum nicht für sich alleine existieren, sondern in einen sozioökonomischen Kontext eingebunden sind.

Deswegen möchte ich an dieser Stelle einige mir wichtige Elemente von TA/TG darstellen und meine Hoffnung zum Ausdruck bringen, dass auch beim BMBF Offenheit für einen Dialog zwischen verschiedenen TA- und ITAKonzepten besteht.

\section{Die Wertediskussion im Feld TA/TG}

„Die dem Menschenglück zugedachte Unterwerfung der Natur hat im Übermaß ihres Erfolges, der sich nun auch auf die Natur des Menschen selbst erstreckt, zur größten Herausforderung geführt, die je dem menschlichen Sein aus eigenem Tun erwachsen ist" (Jonas 1984, S.7). So äußerte sich schon 1984 Hans Jonas im Vorwort zu seinem Buch „Das Prinzip Verantwortung“, in dem er für ein neues, zukunftsorientiertes Verantwortungsbewusstsein im Umgang mit den technischen Möglichkeiten plädierte - und zu der Zeit waren z. B. die heutigen Möglichkeiten der Gentechnik kaum absehbar.

„Angesichts der ins Unermessliche gewachsenen technologischen Macht des Menschen und der Dynamisierung der Lebensumstände in der industriellen Welt sowie angesichts der Gefährdungen von Natur und Kreatur (einschließlich des Menschen selbst) durch Nebenwirkungen des industriellen Prozesses (...) (ist) (...) eine sittliche Erweiterung des Verantwortungskonzepts nötig (...): der Übergang von einer Konzeption der Verursacherverantwortung $\mathrm{zu}$ einer ,Treuhänder"- oder „Heger"-Verantwortung des Menschen, von der rückwirkend zuzuschreibenden Ex-postVerantwortung zur prospektiv ausgerichteten Sorge-für-Verantwortung und Präventionsver- antwortlichkeit, von der vergangenheitsorientierten Handlungsresultatsverantwortung zur zukunftsorientierten, durch Kontrollfähigkeit und Machtverfügbarkeit bestimmten Selbstverantwortung." So gibt Hans Lenk den Inhalt des von Jonas geforderten neuen Verantwortungsbegriffs wieder (Lenk 1988, S. 69 ff.) Lenk macht auch deutlich, dass diese zukunftsorientierte Verantwortung nicht nur dem einzelnen in einer ausschließlich individualistisch orientierten Ethik der moralischen Einzelverpflichtung zukommt, sondern insbesondere als Verpflichtung für kollektiv Handelnde und für „Träger von Verfügungsmacht" einzufordern ist.

An dieser Stelle argumentiert Bode (Bode 2002, S. 53), dass nicht Politik als Funktionalsystem, sondern nur die Person des Politikers oder des Ministers Verantwortung tragen kann und dass hierdurch eine wichtige Verbindung zum Intermediär „Ministerium“ gegeben sei.

Hier fehlt mir dennoch der explizite Anstoß für die Definition einer ethischen Grundhaltung - denn eine Innovations- und Technikanalyse, die Perturbation und Partizipation verschiedener Funktionalsysteme an sich als Erfolg darstellt, ohne zur Bestimmung einer ethischen Grundhaltung anzuregen, greift m. E. zu kurz. Zur Erläuterung dieser Problematik werden im nächsten Kapitel einige Diskussionen zusammenfassend wiedergegeben, die nicht nur in der Ingenieurwissenschaft und von Ingenieuren in der Praxis zu diesem Thema geführt werden.

\section{Moralisch/ethischer Anspruch an Inge- nieur- und Naturwissenschaften}

In der gesellschaftlichen Diskussion werden oft die Ingenieur- und Naturwissenschaften bzw. die entsprechenden Berufsgruppen mit dem Anspruch konfrontiert, als ,kollektiv handeln-

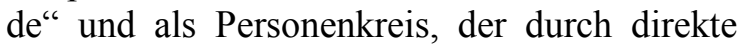
Beteiligung an Forschung und Technikentwicklung Zukunftsgestaltung in besonderer Weise mit beeinflusst, sich der Verantwortungsethik zu stellen und sie zum festen Bestandteil des eigenen Berufs- bzw. Wissenschaftsbildes zu machen.

Es wird eine Technikfolgenabschätzung und daraus folgende Technikgestaltung gefordert, die dazu beitragen sollen, schädliche Ent 
wicklungen $\mathrm{zu}$ vermeiden und zukunftsverträgliche Technologien zu fördern.

Doch die Diskussion darüber, was ,schädlich“ und was ,zukunftsverträglich“" ist, läßt sich nicht durch einige plakative und grundsätzliche ethische „Leitlinien“ beenden, sondern muss in Bezug auf verschiedene Einzeltechnologien im Einzelfall neu und differenziert geführt werden.

Oft geht es darum, zwischen unterschiedlichen Werten und den aus ihnen abzuleitenden Konsequenzen für praktisches Handeln abzuwägen.

Es gibt nur wenige Technologien, wie z. B. die Atombombe, die grundsätzlichen ethischen Forderungen zuwiderlaufen - und auch bei der Atombombe konnten noch im Einzelfall ihrer tatsächlichen historischen Entwicklung und Anwendung „ethisch-moralische“ Argumente für diese Technologie in einem bestimmten politisch-militärischen Umfeld eingeführt werden. Zu den prinzipiell ablehnbaren Technologien gehören sicherlich auch diejenigen Bereiche der Gentechnik, in denen der Versuch unternommen wird, einen „neuen“ Menschen zu schaffen, indem man das genetische Erbmaterial von Menschen so verändert, dass das Produkt nicht mehr genauso „Mensch“ ist, wie wir alle oder in denen Menschen durch Klonen als identische „Massenware" möglichst mit vorhersagbaren Eigenschaften erzeugt werden.

In der komplexen Diskussion über ethische Grundlagen von TA nennt die VDI-Richtlinie zur Technikbewertung (VDI 1991) die folgenden Werte als Maßstäbe für Technikentwicklung und Technikfolgenabschätzung:

Wohlstand, Gesundheit, Sicherheit, Umweltqualität, Persönlichkeitsentfaltung und Gesellschaftsqualität.

Sie macht aber auch deutlich, dass diese Werte im Einzelfall in einer Gesamtbetrachtung abzuwägen sind und dass Entscheidungen einen Ausgleich zwischen aus diesen Werten ableitbaren unterschiedlichen oder gar entgegengesetzten Präjudizierungen erarbeiten sollten.

Dabei wird von der Überlegung ausgegangen, dass moderne Technik wesentlich mitbewirkt hat, die Lebens- und Arbeitsbedingungen vieler Menschen zu verbessern und das durchschnittliche Lebensalter der Menschen, ihre Mobilität und ihre Informations- und Konsummöglichkeiten zu erhöhen.
Umweltschäden, Ressourcenverknappung, die Verringerung von Arbeitsplätzen oder eine gestiegene psychische Belastung vieler Menschen in der Arbeit mit neuen Technologien sind die Kehrseite dieser Entwicklungen.

Bei einer differenzierten Bewertung von Technik geht es einerseits darum, den Januskopf der Technik, die Möglichkeit negativer (Neben-)Wirkungen trotz vielfach positiver Absichten, wirklich wahrzunehmen und nicht einfach abzustreiten. Andererseits sollen die positiven Gestaltungsmöglichkeiten der Technik genutzt werden.

Auf diesen Anspruch hin wird in den Ingenieur- und Naturwissenschaften oft mit zwei Argumentationsmustern reagiert:

\section{Argumentationsmuster 1: Technikentwicklung ist ein komplexer Prozess - und deswegen sind einfache ethische Maßstäbe und daraus resultierende konkrete Forderungen nicht an- wendbar.}

Weder die Ingenieur- noch die Naturwissenschaften sind heute in der Lage, die Chancen und Risiken der technologischen Entwicklung umfassend $\mathrm{zu}$ überblicken. Für viele Wissenschaftler/innen gibt es wegen ihrer eigenen Spezialisierung und wegen der raschen weltweiten Technologieentwicklung große Schwierigkeiten, die Veränderungen in den über ihr eigenes Fachgebiet hinausgehenden Bereichen bewusst wahrzunehmen. Gleichzeitig steigt der Einfluss der technischen Neuerungen auf Wirtschaft und Industrie, aber auch auf die konkreten Lebensund Arbeitsverhältnisse der Menschen und auf die Natur. Dabei nimmt die Vernetzung technischer, sozialer, ökonomischer und ökologischer Entwicklungen zu, so dass Kausalketten-basierte Extrapolationen in die Zukunft oft darunter leiden, Vernetzungen und Wechselwirkungen unterschiedlichster Einflussgrößen nicht genügend berücksichtigt zu haben.

Man hat es

„bei der Technikentwicklung in aller Regel
mit einem langen, über viele Jahre und Jahr-
zehnte reichenden vielschichtigen Prozess zu
tun, der irgendwo in der Grundlagenforschung
einsetzt und über eine Vielzahl von Entwick-
lungsschritten irgendwann in einer Technolo-
gie seine Realisierung findet. Von dieser
,Technologie ist es dann zumeist noch einmal


ein weiter, komplexer und von vielen kognitiven, ökonomischen und anderen Mechanismen gesteuerter Prozess mit einer großen Zahl unbegangener Seitenwege und möglicher Sackgassen bis zur Entstehung konkreter technischer Systeme und Gegenstände, deren praktische Nutzung überhaupt erst relevante Folgen zeigen kann“" (BMBF 1989, S.9).

Deswegen könne der Forderung nach ,ethischer Verantwortung" nur sehr schwer nachgekommen werden, da Zukunftsfolgen und Nebenwirkungen der eigenen Tätigkeit gar nicht abzuschätzen seien.

Argumentationsmuster 2: Dem Risiko des Tuns steht ein Risiko des Unterlassens gegenüber erst die Chancen debattieren, dann die Risiken.

TA wird in vielen gesellschaftlichen und politischen Diskussionen oft erst oder insbesondere dann gefordert, wenn negative Folgen neuer Technologien deutlich werden. Krasse Beispiele sind durch Medikamente hervorgerufene Missbildungen bei Kindern oder Gesundheitsbeeinträchtigungen durch Holzschutzmittel in Wohnräumen. In einer technikkritischen TA-Diskussion stehen also die Risiken der Umsetzung und Anwendung bestimmter Technologien im Vordergrund. In vielen ingenieurwissenschaftlichen Debatten werden dagegen die Chancen der Anwendung neuer Technologien und die Risiken der Unterlassung bestimmter technologischer Forschung und Entwicklung in den Vordergrund gestellt. Nimmt man wieder das Beispiel der Kernenergie, so wird in den Ingenieurwissenschaften oft das Argument der Vorbildfunktion Deutschlands hervorgehoben, da es hier - gerade durch den breiten Widerstand der AntiAKW-Bewegung - eine tiefgehende Risikobetrachtung und eine entsprechende Sicherheitsund Störfall-Verhütungskompetenz gibt, die in vielen anderen Ländern fehlt. Unterläßt nun Deutschland weitere öffentlich und privat finanzierte Forschung und Entwicklung in diesem Bereich wegen des erkannten langfristigen Risikos der Kernenergie und steigt konsequent aus der Kernenergienutzung aus, dann kann ein unerwünschter Nebeneffekt die weltweite Senkung von Sicherheitsstandards sein - also auch Unterlassen hat seine Risiken.

Wenn auch die Kritiker hier einwenden, das gelte umgekehrt auch für das Unterlassen einer ähnlich der Kernenergie finanziell geförderten Forschung und praxisorientierten Entwicklung im Bereich Sonnen- und Windenergie in den letzten 40 Jahren, so wird dadurch das grundlegende Argument nicht widerlegt, sondern eher bestätigt: Dem Risiko des Tuns steht ein Risiko des Unterlassens gegenüber.

Beide Argumente können zu einer Bereicherung der TA-Diskussion in den nichtingenieurwissenschaftlichen Debatten beitragen: Wenn die Komplexität des Entstehensprozesses neuer Technologien ernst genommen und die Risiken der Unterlassung ebenso abgeschätzt werden wie die Risiken der Umsetzung neuer Technologien, ist das keine Aufweichung von TA, sondern eine Komplettierung der Methodik.

Andererseits muss aber davor gewarnt werden, diese beiden Argumente dazu zu nutzen, TA grundsätzlich abzulehnen, es wegen methodischer Schwierigkeiten gar nicht zu betreiben oder wenn, dann ethisch/moralische Grundpositionen gar nicht oder nur am Rande zu benennen, eine Versuchung, der - wie das Beispiel Bodes zeigt - nicht nur Ingenieur/ innen in Wissenschaft und Industrie oft ausgesetzt sind.

\section{ITA - Innovationsorientierte Technikfol- genabschätzung, Technikbewertung und Technikgestaltung - als TA-Modell in der Diskussion des AKTAB NRW}

Im AKTAB, dem Arbeitskreis Technikfolgenabschätzung und Bewertung des Landes Nordrhein-Westfalen (Henning et al. 1999), einem interdisziplinär zusammengesetzten TA-Arbeitskreis, aus dessen Arbeit u. a. das auch von Bode zitierte „TA-Handbuch“ (Bröchler et al. 1999) entstanden ist, wurde die im Folgenden kurz beschriebene „ITA“ - ,innovationsorientierte TA" - sowohl als ingenieurwissenschaftliches als auch als sozialwissenschaftliches TA-Modell relativ breit diskutiert.

Die ingenieurwissenschaftliche Interpretation dieses TA-Modells ist durch die folgenden drei Schritte gekennzeichnet (FuchsFrohnhofen und Henning 1999):

Erstens: Bei allen neuen technischen Entwicklungen sollen, in mehr oder weniger systematischer Form, die möglichen Folgen der neuen Technologien in verschiedenen Bereichen abgeschätzt werden (z. B. Ökologie, Ge 
sellschaft, Datenschutz, möglicher Verkaufserfolg, Arbeitsplätze etc.). Dabei kann in der Regel nicht eine Einzeltechnologie alleiniger Ausgangspunkt sein, sondern es muss vielmehr ein Set von Technik, Organisation und Personal auf mögliche Folgen hin abgeschätzt werden, also die Technik im Zusammenhang ihrer künftigen organisatorisch/ökonomischen Einbettung und ihres Nutzungszusammenhangs durch den Menschen betrachtet werden.

Zweitens müssen die möglichen Folgen auf der Basis der vorhandenen Wertmaßstäbe beurteilt werden. Dabei sind sicherlich betriebliche Werte, menschliche Grundwerte und gesellschaftliche Wertvorstellungen zum Ausgleich zu bringen, um eine Basis für zukunftsfähige Entscheidungen $\mathrm{zu}$ bekommen. Einen guten methodischen Ausgangspunkt bietet hierzu die VDI-Richtlinie 3780.

Drittens geht es bei ITA immer darum, auf der Basis von Folgenabschätzung und Beurteilung zu der Erarbeitung von Handlungs- und Gestaltungsoptionen zu kommen.

Innovationsorientierte TA wird - eingebunden in einen komplexen Prozess der Technikentwicklung - nicht den ,einen besten Weg" herausstellen wollen oder eine einzelne Zukunftsoptionen positiv oder negativ bewerten. Für das Gelingen innovationsorientierter TA ist es unabdingbar, mehrere Optionen oder Varianten künftiger Entwicklungen zu diskutieren oder unterschiedliche Innovationsvarianten erst zu generieren. Erst im Vergleich und in der Abwägung zwischen diesen Varianten und Zukunftsoptionen und in der Erörterung der Frage „Kann man das nicht auch anders machen? Welche Alternativen gibt es?" konkretisiert sich der Innovationsgedanke von TA in den Ingenieurwissenschaften.

Eine solche innovationsorientierte TA kann als ingenieurwissenschaftliches Instrumentarium dazu beitragen, neue Technologien zukunftsund wertorientierter zu gestalten. Dazu gehört natürlich auch, bei der Bewertung von Optionen zukünftiger Entwicklungen dort Einspruch zu erheben, wo sich negative Folgen abzeichnen.

In den meisten Fällen aber wird nach Folgenabschätzung und Bewertung die zukunftsorientierte Gestaltung und Umsetzung positiver Alternativen am Endpunkt eines ingenieurwissenschaftlichen Prozesses innovationsorientierter TA stehen.
Neben dieser Beschreibung von „ITA“ aus Sicht von Ingenieur/innen wurde im AKTAB auch ein stärker sozialwissenschaftlich geprägtes ITA-Modell erarbeitet (Steinmüller et al. 1999). Auch in diesem Modell wird ITA als Verfahren zur Mitgestaltung von Innovationsprozessen bezeichnet, bleibt also nicht bei Technik- und Innovationsanalyse aus sozialwissenschaftlicher Sicht stehen, sondern zieht den Aspekt der Mitwirkung an Technikgestaltung mit ein. Mit dem Konzept von Bode verbindet diesen Ansatz, dass sich „ITA nicht als Lieferant von neutralem Entscheidungswissen" (Steinmüller et al. 1999, S. 133) versteht, sondern als eine Moderationsinstanz im Rahmen des Innovationsprozesses. Hierbei dient das „Objektinterview" (Tschiedel 1999) als Mittel zur methodischen Sicherung und Beteiligungsorientierung.

\section{Abschließende Bemerkungen}

Schaut man sich die deutsche TA-Landschaft 2003 an, so sind einige negative Trends auszumachen.

- Mit der Schließung der TA-Akademie in Baden-Württemberg und der Einstellung der Förderung für den $\mathrm{AKTAB}$ in NRW, der sich daraufhin als Arbeitskreis des Landes aufgelöst hat, wird deutlich: von maßgebenden Personen in der Politik wird TA oder ITA als ,nice to have" betrachtet, das man sich in finanziell guten Zeiten leisten kann, das man aber bei knapper werdenden Finanzmitteln recht schnell in einer passenden Streichliste unterbringt.

- Die Konkurrenz um begrenzte Fördermittel, räumliche und institutionelle Trennungen, politisch oder konzeptionell unterschiedliche Ansatzpunkte, immer noch vorhandene Unfähigkeiten zur Interdisziplinarität und ein Wechselspiel von persönlichen Sympathien und Antipathien haben zu konkurrierenden und unabgestimmten Diskursen in der TA-Landschaft geführt- wie sich auch an den allein in diesem Beitrag benannten drei „ITA“-Varianten zeigt.

Es würde mich freuen, wenn das BMBF - neben der sicherlich richtigen Durchführung der von Bode beschriebenen ITA-Projekte - diese Entwicklungen zum Anlass nimmt, die Lage der 
gesamten TA-Landschaft in Deutschland zu analysieren $^{2}$, die wesentlichen Akteure zu gemeinsamen Diskursen zusammenzubringen und davon ausgehend, der gesamten TA-Community die Chance zu geben, sich eine neue realistische Zukunftsperspektive zu erarbeiten.

\section{Anmerkungen}

1) Gerade Akteure der Zivilgesellschaft haben ja in der Geschichte der TA immer wieder den nötigen politischen Druck erzeugt, um TA zu institutionalisieren und zu finanzieren - und zwar indem sie die kommunikativen Grenzen einzelner Funktionalsysteme überwunden und eine funktionalsystem-übergreifende gesellschaftliche Diskussion initiiert haben - oder wie ist aus systemtheoretischer Perspektive erklärbar, dass z. B. bayrische Bäuerinnen in Wackersdorf Vertretern der Funktionalsysteme Wissenschaft, Wirtschaft und Politik überzeugende und verstandene Argumente zur Funktionsweise und zu technischen, ökologischen und ökonomischen Risiken von Wiederaufarbeitungsanlagen entgegensetzen konnten?

2) Und zwar möglichst unvoreingenommen, ohne den eigenen ITA-Ansatz als ,modern“ und die anderen existierenden TA/TG oder ITA-Konzepte als „historische Vorgängermodelle“ und damit letztlich als überholt zu kennzeichnen.

\section{Literatur}

Bode, O., 2002: Die ITA der Gesellschaft - Praxisbeobachtungen zur Innovations- und Technikanalyse auf der Grundlage der Theorie sozialer Systeme. In: Development and Perspectives, No. 2. Göttingen, S. 35-68

Bröchler, S., Simonis, G., Sundermann, K. (Hrsg.), 1999: Handbuch Technikfolgenabschätzung. Berlin (Band 1-3)

BMBF - Der Bundesminister für Forschung und Technologie, 1989: Memorandum zur Technikfolgenabschätzung. Bonn

Fuchs-Frohnhofen, P., Henning, K., 1999: TAVerständnis in den Ingenieurwissenschaften. In: Bröchler, S. u. a., S. 65-72

Henning, K, Fuchs-Frohnhofen, P., Olbertz, E., 1999: Der AKTAB NRW. In: Bröchler, S. u. a., S. $451-458$

Jonas, H., 1984: Das Prinzip Verantwortung. Versuch einer Ethik für die technologische Zivilisation. Frankfurt: Suhrkamp-Verlag
Lenk, H., 1988: Verantwortung in, für, durch Technik. In: Lenk, H.; Bungard, W. (Hrsg.): Technikbewertung - Philosophische und psychologische Perspektiven. Frankfurt: Suhrkamp-Verlag, S. 69-87

Steinmüller, K-H., Tacke,K., Tschiedel, R., 1999: Innovationsorientierte Technikfolgenabschätzung. In: Bröchler, S. u. a., S. 129-146

Tschiedel, R., 1999: Objektinterview. In: Bröchler, S. u. a., S. 687-696

VDI - Verein Deutscher Ingenieure, 1991: Technikbewertung - Begriffe und Grundlagen (VDIRichtlinie 3780). Düsseldorf

\section{Kontakt}

Dr.-Ing. Paul Fuchs-Frohnhofen

Karl-Carstens-Strasse 1

52146 Würselen/Aachener Kreuz

Tel.: + 49 (0) 2405 / 4552 - 0

Fax: + 49 (0) $2405 / 4552$ - 200

E-Mail: paul.fuchs-frohnhofen@mat-gmbh.de

Internet: http://www.mat-gmbh.de

\section{Innovations- und Technikanalyse durch Hochschulbildung stärken}

\section{von Bernd Steffensen, Fachhochschule Darmstadt}

I. Die Geschichte der Technikfolgenabschätzung (TA) ist wechselhaft. Eindrucksvoll zeigt dies der sehr persönliche Bericht von Josef Bugl (1994). Bugl gehört zu den Personen in der Bundesrepublik Deutschland, die aufgrund ihres (beruflichen) Werdegangs - Politiker und Vertreter der Wirtschaft - in besonderer Weise geeignet scheinen, die Ziele der nun als Innovations- und Technikanalyse bezeichneten systematischen Bewertung von Chancen und Risiken neuer Technologien voranzutreiben. In diesem Sinne ist sein langjähriges Engagement bei der Initiierung und Gründung, beim Aufbau sowie bei der Konsolidierung der nun vor dem endgültigen Aus stehenden Akademie für Technikfolgenabschätzung in Baden-Württemberg zu verstehen und zu würdigen. Selbst habe ich 19922000 am Aufbau des Bereiches Technik, Orga 